\title{
Stem Cell Therapy for Intestinal Ischemia- Reperfusion: Current State and Perspectives
}

\author{
Audelia Eshel Fuhrer²*, Ben-Shahar Yoav², Kopplemann Tal'2, Haham Alon ${ }^{1,3}$ and Igor Sukhotnik ${ }^{1,2}$ \\ ${ }^{1}$ Sackler Faculty of Medicine Tel Aviv University, Israel \\ ${ }^{2}$ Department of Pediatric Surgery, Dana-Dwek Children's Hospital, Tel Aviv Sourasky Medical Center, Israel \\ ${ }^{3}$ Department of Neonatology, Tel Aviv Sourasky Medical Center, Dana-Dwek Children's Hospital, Israel
}

Submission: October 17, 2019; Published: November 12, 2019

*Corresponding author: Audelia Eshel Fuhrer, Department of Pediatric Surgery, Dana-Dwek Children’s Hospital, Tel Aviv Sourasky Medical Center, Tel Aviv, Israel

\begin{abstract}
Stem cell therapy has received significant attention as a novel therapeutic approach to restore intestinal function after ischemia-reperfusion (IR) injury. Necrotizing enterocolitis (NEC) is a catastrophic disease caused by a variety of factors occurring mostly in preterm infants. Although being extensively investigated in the past two decades, there have been no significant advancements in the management of this disease, emphasizing the urge for a novel therapeutic pathway. IR model of intestinal injury has been widely used as an indirect model of NEC, usually conducted by temporary occlusion of the intestinal blood supply. This review aims to illustrate the role of stem cell therapy in promotion of intestinal recovery following IR. Among the many identified stem cells to date, it is likely that mesenchymal and amniotic stem cells would be the most useful and practical cell-based therapies for this purpose. Using acellular components such as exosomes seems a promising technique as well. Future directions of cellular therapy in intestinal IR are currently being explored and might help stimulate future studies on the role of cellular therapy in NEC.
\end{abstract}

Keywords: Intestinal; Ischemia-Reperfusion (IR); Necrotizing Enterocolitis (NEC); Stem cell

Abbreviations: IR: Ischemia-Reperfusion; NEC: Necrotizing Enterocolitis; ROS: Radical Oxygen Species; ISCS: Intestinal Stem Cells; AISCS: Actively Proliferating ISC; RISCS: Reserve ISC; BMP: Bone Morphogenetic Protein; MSCS: Mesenchymal Stem Cell; USCS: Umbilical-Derived Mesenchymal Stromal Cells; AFSS: Amniotic Fluid-Derived Stem Cells

\section{Introduction}

Intestinal ischemia-reperfusion (IR) injury remains a clinically challenging problem despite decades of research in this area. Causes of mesenteric ischemia such as necrotizing enterocolitis (NEC), midgut volvulus or incarcerated hernia and its major complication of gangrenous necrosis of portions of the gastrointestinal tract are well recognized by all pediatric surgeons [1]. Common to most critically ill patients the preservation of blood flow to vital organs occurs at the expense of blood flow to the intestine. Restoration of blood flow following intestinal ischemia is necessary to maintain cell function and viability; however, the reintroduction of oxygen can initiate a cascade of events that exacerbates intestinal damage through formation of radical oxygen species (ROS) and changes in lipid mediator synthesis [2]. The increased production of ROS, combined with decreased perfusion, contributes to endothelium damage [3], increased epithelial permeability [4], and gut barrier dysfunction leading to bacteria and bacterial products translocation into the systemic circulation. The dynamic process of enterocyte turnover following intestinal IR is mediated by the rates of crypt cell proliferation, cell migration along crypt-villus axis, and cell death via apoptosis channel. Intestinal stem cells (ISCs) are fundamental cornerstones in gut biology, ensuring homeostatic turn-over of intestinal epithelial cells and presenting a reserved number of cells that can be stimulated after tissue injury (such as IR) [5]. In addition to their ability to self-renew, ISCs differentiate into specialized cell populations which are confined to the crypt base and play a critical role in tissue maintenance and regeneration. Growing evidence points out into two ISCs populations: actively proliferating ISC (aISCs) and quiescent ISC thought to be activated upon injury and to act as reserve ISC (rICSs) [6]. Two hypotheses exist regarding the exact identity and location of the ISCs populations: the "4" position model counting from the bottom of the crypt directly above the Paneth cells, and the zone model identified as the crypt base columnar (CBC) cells between the Paneth cells. Experimental IR models, induced by vascular occlusion, demonstrated injury expressed as sloughing of epithelium at the villus tip progressing 
towards the crypt base, with an architectural preservation of the crypts [7]. These findings stand in line with the theory that following tissue insult the potent regeneration area is preserved, as the crypts are assumed to inhabit the ISCs. Nevertheless, every ICS population has probably a different role in this process; using a porcine model, Gonzalez et al. [8] have recently demonstrated that following 3-4h of ischemic injury aISCs underwent apoptosis, whereas rISCs remained preserved. Moreover, these rISCs were retained in ex vivo culture and appeared to provide a proliferative pool of cells during the recovery period [8].

Various signaling cascades have been studied in the field of intestinal IR injury. These cascades are all involved in the control of ISC activity, proliferation, lineage commitment, terminal differentiation, and cell survival during tissue regeneration following IR. They are also involved in normal gut development. These signaling pathways include Wnt/ $\beta$-catenin, Hedgehog, Bone morphogenetic protein (BMP) and Notch [9]. Our research group has demonstrated that $24 \mathrm{~h}$ following IR, Hedgehog [10] and BMP [11] signaling pathways were inhibited, which correlated with inhibited cell turnover. Signs of intestinal recovery appeared $48 \mathrm{~h}$ after IR and were then correlated with activated Hedgehog and BMP signaling pathways. A different study has demonstrated that Notch signaling was inhibited $48 \mathrm{~h}$ following intestinal IR injury [12]. These findings suggest that at the first phase of IR recovery intestinal stem cells differentiate towards secretory progenitors rather than absorptive cells. In a recent study, Li et al., [13] have shown that during experimental NEC, impaired ISCs activity was associated with inhibited Wnt/ $\beta$-catenin signaling and impaired intestinal regeneration [13].

\section{Discussion}

In line with these findings, stem cell therapy has become a novel strategy for the treatment of intestinal IR. The ability of ISC to self-replicate, differentiate, prevent apoptosis and reduce inflammation has raised interest in the potential therapeutic value of these cells in IR and NEC. Jiang et al. have shown that intestinal treatment with mesenchymal stem cell (MSCs) decreases the severity of intestinal barrier injury and bacterial translocation and lowers the levels of serum lactate in a rat model of intestinal IR [14]. Umbilical-derived mesenchymal stromal cells (USCs) are isolated from the umbilical cord and are considered multipotent. USCs have been used successfully in animal models of IR and NEC [15]. Using human USCs, Jensen et al. [16] demonstrated that intraperitoneal USC therapy following intestinal IR injury improved mesenteric perfusion, prevented intestinal mucosal damage, and increased the production of certain inflammatory mediators associated with cell injury [16]. Amniotic fluidderived stem cells (AFSs) can be cultured from amniotic fluid samples collected during routine amniocentesis or at planned caesarean section. Intraperitoneal injection of these cells has been shown to significantly reduce the incidence and severity of NEC, decrease histological signs of injury and lead to improved gut barrier function during experimental NEC [17, 18]. It has been discovered that certain cells contain organoids that secrete growth factor-containing vesicles called exosomes. These vesicles bind to target cells at the extracellular space and can manipulate signaling pathways or unload their contents into the target cells' cytoplasm [19]. Nowadays, intestinal epithelial organoids can be cultured from intestinal crypts and have been recently used to study NEC progression in search for novel therapeutic treatments. Li et al. [13] used mice and human models to provoke NEC induced disruption of the intestinal Wnt-ISC-regeneration axis [13]. The authors have shown that NEC-derived organoids were unable to maintain intestinal homeostasis. However, exogenous Wht administration restored the organoids capability of intestinal homeostasis, supporting the role of exogenous Wnt as novel therapeutic pathway. A recent promising study in rats has demonstrated the feasibility of creating acellular natural matrix without disruption of villus-crypt architecture and mechanical characteristics which may represent an innovative platform for small bowel bioengineering for usage as an alternative approach to experimental animal models or even for allogeneic intestinal transplantation [20].

\section{Conclusion}

In conclusion, although the mechanisms of ISCs in gut IR are still not fully understood, stem cell therapy is considered to represent a promising range of novel therapeutic strategies for intestinal IR and NEC. To the best of our knowledge, it is likely that AFSs or MSCs would be the most useful and practical cell-based therapies for these conditions. Acellular therapies using exosomes for example are yet to be defined but seem to carry some promise. Further clinical research is prompted in order to fully understand the downstream effects of intestinal cells differentiation and regeneration in NEC.

\section{References}

1. Carden DL, Granger DN (2000) Pathophysiology of ischaemia-reperfusion injury. J Pathol 190(3): 255-266.

1. Kurose I, Argenbright LW, Wolf R, Lianxi L, Granger DN (1997) Ischemia/reperfusion-induced microvascular dysfunction: role of oxidants and lipid mediators. Am J Physiol 272((6): 2976-2982.

2. Wolin MS (2000) Interactions of oxidants with vascular signaling systems. Arterioscler Thromb Vasc Biol 20(6): 1430-1442.

3. Mangino JE, Kotadia B, Mangino MJ (1996) Characterization of hypothermic intestinal ischemia-reperfusion injury in dogs. Effects of glycine. Transplantation 62(2): 173-178.

4. Bjerknes M, Cheng H (1981) The stem-cell zone of the small intestinal epithelium: III. Evidence from columnar, enteroendocrine, and mucous cells in the adult mouse. Am J Anat 160(1): 77-91.

5. Yan KS, Chia LA, Li X, Ootani A, Su J, et.al. (2012) The intestinal stem cell markers Bmi1 and Lgr5 identify two functionally distinct populations. Proc Natl Acad Sci USA 109(2): 466-471.

6. Itoh H, Yagi M, Hasebe K, Fushida S, Tani T, et.al. (2002) Regeneration of small intestinal mucosa after acute ischemia-reperfusion injury. Dig Dis Sci 47(12): 2704-2710.

7. Gonzalez LM, Stewart AS, Freund J, Kucera CR, Dekaney CM, et.al. (2019) Preservation of reserve intestinal epithelial stem cells following severe ischemic injury. Am J Physiol Gastrointest Liver Physiol 316(4): 482-494. 
8. De Santa Barbara P, van den Brink GR, Roberts DJ (2003) Development and differentiation of the intestinal epithelium. Cell Mol Life Sci 60(7): 1322-1323.

9. Ben-Shahar Y, Pollak Y, Bitterman A, Coran AG, Bejar IN, et al. (2019) Sonic hedgehog signaling controls gut epithelium homeostasis following intestinal ischemia-reperfusion in a rat. Pediatr Surg Int 35(2): 255-261.

10. Ben-Shahar Y, Abassi Z, Kreizman-Shefer H, Pollak Y, Bhattacharya U, Sukhotnik I (2019) Accelerated intestinal cell turnover correlates with stimulating BMP signaling cascaded following intestinal ischemiareperfusion in a rat. Eur J Pediatr Surg.

11. Ben-Shahar Y, Abassi Z, Pollak Y, Bitterman A, Kreizman-Shefer H, et.al. (2019) Accelerated cell turnover $48 \mathrm{~h}$ after intestinal ischemia is NOTCH independent. Pediatr Surg Int.

12. Li B, Lee C, Cadete M, Zhu H, Koike Y, et.al. (2019) Impaired Wnt/ $\beta$-catenin pathway leads to dysfunction of intestinal regeneration during necrotizing enterocolitis. Cell Death Dis 10(10):743

13. Jiang H, Qu L, Li Y, Gu L, Shi Y, et.al. (2011) Bone Marrow Mesenchymal Stem Cells Reduce Intestinal Ischemia/Reperfusion Injuries in Rats. J Surg Res 168(1): 127-134.

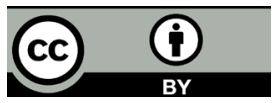

This work is licensed under Creative Commons Attribution 4.0 Licens DOI:_10.19080/AJPN.2019.08.555792
14. Jensen AR, Manning MM, Khaneki S, Drucker NA, Markel TA (2016) Harvest tissue source does not alter the protective power of stromal cell therapy after intestinal ischemia and reperfusion injury. J Surg Res 204(2): 361-370

15. Jensen AR, Drucker NA, Ferkowicz MJ, Markel TA (2018) Umbilical mesenchymal stromal cells provide intestinal protection through nitric oxide dependent pathways. J Surg Res 224: 148-155.

16. Zani A, Cananzi M, Fascetti-Leon F, Lauriti G, Smith VV, et.al. (2014) Amniotic fluid stem cells improve survival and enhance repair of damaged intestine in necrotising enterocolitis via a COX-2 dependent mechanism. Gut 63(2): 300-309.

17. McCulloh CJ, Olson JK, Wang Y, Vu J, Gartner S, Besner GE (2017) Evaluating the efficacy of different types of stem cells in preserving gut barrier function in necrotizing enterocolitis. J Surg Res 214: 278-285.

18. Goodarzi P, Larijani B, Alavi-Moghadam S, Tayanloo-Beik A, Mohamadi-Jahani F, et.al. (2018) Mesenchymal Stem Cells-Derived Exosomes for Wound Regeneration. Adv Exp Med Biol 1119: 119-131.

19. Totonelli G, Maghsoudlou P, Garriboli M, Riegler J, Orlando G, et.al. (2012) A rat decellularized small bowel scaffold that preserves villus-crypt architecture for intestinal regeneration. Biomaterials 33(12): 3401-3410.

\section{Your next submission with Juniper Publishers will reach you the below assets}

- Quality Editorial service

- Swift Peer Review

- Reprints availability

- E-prints Service

- Manuscript Podcast for convenient understanding

- Global attainment for your research

- Manuscript accessibility in different formats

( Pdf, E-pub, Full Text, Audio)

- Unceasing customer service

Track the below URL for one-step submission https://juniperpublishers.com/online-submission.php 